Louisiana State University

LSU Digital Commons

3-1-2014

\title{
Stellar diameters and temperatures. IV. Predicting stellar angular diameters
}

Tabetha S. Boyajian

Yale University

Gerard Van Belle

Lowell Observatory

Kaspar Von Braun

Max Planck Institute for Astronomy

Follow this and additional works at: https://digitalcommons.Isu.edu/physics_astronomy_pubs

\section{Recommended Citation}

Boyajian, T., Van Belle, G., \& Von Braun, K. (2014). Stellar diameters and temperatures. IV. Predicting stellar angular diameters. Astronomical Journal, 147 (3) https://doi.org/10.1088/0004-6256/147/3/47

This Article is brought to you for free and open access by the Department of Physics \& Astronomy at LSU Digital Commons. It has been accepted for inclusion in Faculty Publications by an authorized administrator of LSU Digital Commons. For more information, please contact ir@lsu.edu. 


\title{
STELLAR DIAMETERS AND TEMPERATURES. IV. PREDICTING STELLAR ANGULAR DIAMETERS
}

\author{
Tabetha S. Boyajian ${ }^{1}$, Gerard van Belle ${ }^{2}$, and Kaspar von Braun ${ }^{3}$ \\ ${ }^{1}$ Department of Astronomy, Yale University, New Haven, CT 06511, USA; tabetha.boyajian@yale.edu \\ ${ }^{2}$ Lowell Observatory, Flagstaff, AZ 86001, USA \\ ${ }^{3}$ Max Planck Institute for Astronomy, Königstuhl 17, D-69117 Heidelberg, Germany \\ Received 2013 August 7; accepted 2013 November 17; published 2014 January 21
}

\begin{abstract}
The number of stellar angular diameter measurements has greatly increased over the past few years due to innovations and developments in the field of long baseline optical interferometry. We use a collection of high-precision angular diameter measurements for nearby, main-sequence stars to develop empirical relations that allow the prediction of stellar angular sizes as a function of observed photometric color. These relations are presented for a combination of 48 broadband color indices. We empirically show for the first time a dependence on metallicity of these relations using Johnson $(B-V)$ and Sloan $(g-r)$ colors. Our relations are capable of predicting diameters with a random error of less than $5 \%$ and represent the most robust and empirical determinations of stellar angular sizes to date.
\end{abstract}

Key words: Hertzsprung-Russell and C-M diagrams - planetary systems - stars: early-type stars: fundamental parameters - stars: general - stars: late-type

Online-only material: color figures

\section{INTRODUCTION}

Empirical determination of stellar radius provides essential constraints in a variety of fields within astrophysics. For instance, stellar models or indeed any calibration equations that involve stellar radii have to be able to reproduce stellar radius measurements based on, e.g., interferometry or the study of eclipsing binary stars (Torres et al. 2010). Exoplanetary parameters are functions of stellar astrophysical parameters (von Braun et al. 2012). Any ability to reliably predict stellar sizes would be of great use for the study of microlensing events (Calchi Novati et al. 2010), searches for Kuiper Belt Objects (KBOs; Wang et al. 2010), asteroseismology (Huber et al. 2012), binary stars (Southworth et al. 2005), exoplanet transits (Assef et al. 2009), and many more.

It has long been known that there exists a relationship between stellar surface brightness and stellar broadband colors (Wesselink 1969). From the Stephan-Boltzmann law, $L \propto$ $R^{2} T_{\text {eff }}^{4}$, it can also be established that surface brightness is dependent on apparent magnitude and stellar angular size (e.g., see the discussion in the introduction of Barnes \& Evans 1976). These two relations can be combined to show that stellar angular size can be predicted on the basis of multicolor photometry. Stellar color-based on two bands-can be calibrated to produce an "absolute" (constant distance) angular size, i.e., the angular size that a star would have if it were moved to a distance at which its apparent magnitude were zero. The apparent stellar magnitude in one of the two bands can then be used to provide the scale between that "zeromagnitude" angular size and the measured angular size at its actual distance. Barnes \& Evans (1976) demonstrate the gross insensitivity of these techniques to interstellar reddening, greatly extending their general applicability throughout astrophysics, e.g., for calibration of Cepheid period-luminosity relations (di Benedetto 1995; Fouque \& Gieren 1997; Kervella et al. 2004a; Groenewegen 2007; Storm et al. 2011a, 2011b) or distances to eclipsing binaries (Lacy 1977; Southworth et al. 2005).

Since stellar apparent magnitudes are direct observables, the methods described above are simple in their application once they are calibrated. Until recently, however, the key missing element was a sufficient body of data with which to carry out those empirical calibrations. Stellar photometry was available, but empirically measured stellar angular sizes were not. Optical interferometry provides the most direct measures of stellar angular diameters-where only the transformation from uniform disk to limb-darkened angular diameter relies on model calculations. Interferometry has provided angular diameters for hundreds of evolved stars over the past $15 \mathrm{yr}$ (e.g., van Belle et al. 1999; Mozurkewich et al. 2003), but similarly sized homogeneous data sets for main-sequence stars have only become available in the last few years with our concerted CHARA Array program (Boyajian et al. 2008, 2009, 2012a, 2012b, 2013).

This paper provides an updated account of, as well as an extension to, empirically derived surface brightness calibrations presented in earlier work (Hindsley \& Bell 1989; di Benedetto 1998; van Belle 1999; Mozurkewich et al. 2003; Kervella et al. 2004b; Di Benedetto 2005), specifically using the nonlinear construction of the functions employed within Bonneau et al. (2006) and Kervella \& Fouqué (2008). Section 2 describes the sample and input data, and Section 3 presents our results with a discussion on their applications. We summarize and conclude in Section 4.

\section{DATA AND ANALYSIS}

\subsection{Definitions}

Data for our analysis are taken from the compilation of angular diameters, broadband photometry, and metallicities presented in Boyajian et al. (2012b, 2013). This anthology of angular diameter measurements comes from an assortment of interferometers: the CHARA Array (Baines et al. 2008, 2012; Boyajian et al. 2012a; Ligi et al. 2012; Di Folco et al. 2004; Bigot et al. 2011, 2006; von Braun et al. 2011; Crepp et al. 2012; Bazot et al. 2011; Huber et al. 2012), the Palomar Testbed Interferometer (van Belle \& von Braun 2009), the Very Large Telescope Interferometer (Kervella et al. 2003a, 2003b, 2004c; Di Folco et al. 2004; Thévenin et al. 2005; Chiavassa et al. 2012), 


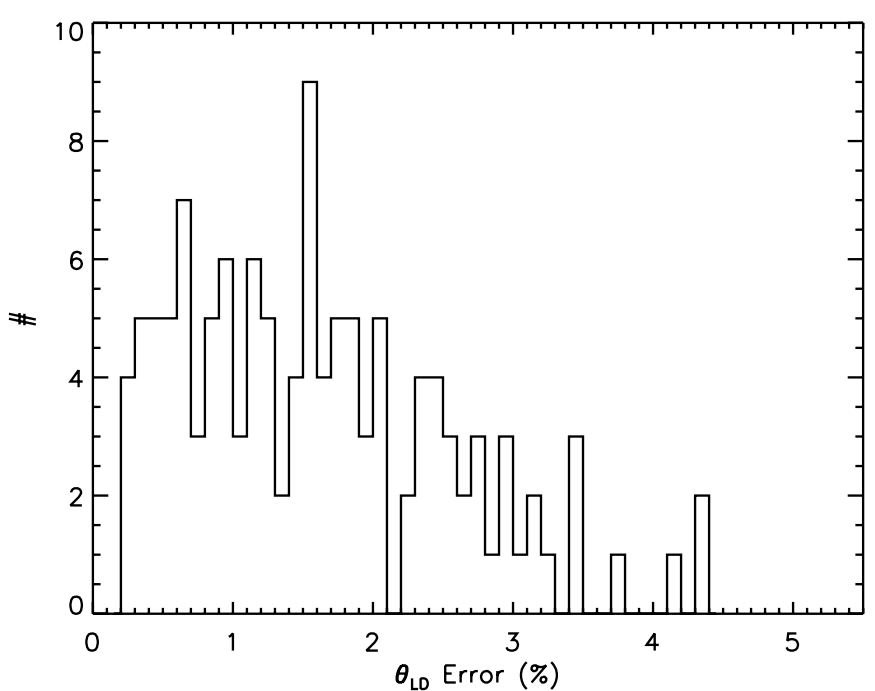

Figure 1. Histogram showing the distribution of the angular diameter errors for stars used in this sample; see Section 2.1 for details.

the Sydney University Stellar Interferometer (Davis et al. 2011), the Narrabri Stellar Intensity Interferometer (Hanbury Brown et al. 1974b), Mark III (Mozurkewich et al. 2003), and the Navy Prototype Optical Interferometer (Nordgren et al. 1999, 2001). The interferometer measures a uniform disk angular diameter, which is transformed into a limb-darkened angular diameter using calculated coefficients from model atmospheres (e.g., Claret 2000) that depend on the wavelength of the observation as well as the stellar photospheric properties. The majority of the observations were made in the infrared, where the correction from uniform disk to limb-darkened diameter is small, $\sim 2 \%-3 \%$, and the errors in the correction for limbdarkening contribute $\ll 0.1 \%$ to the total angular diameter error budget. We impose a limit to only include objects with angular diameters measured to better than $5 \%$. The resulting sample has errors spanning a range of $0.2 \%-4.3 \%$ with a median error of $1.5 \%$. This distribution of angular diameter errors is plotted in Figure 1.

Boyajian et al. (2013) use the interferometric sample described above to build an assortment of color-temperature relations. For our analysis, we use the broadband photometry measurements compiled in Boyajian et al. (2013) from the Johnson $\left(B V R_{\mathrm{J}} I_{\mathrm{J}} J H K\right)$, Cousins $\left(R_{\mathrm{C}} I_{\mathrm{C}}\right)$, Kron $\left(R_{\mathrm{K}} I_{\mathrm{K}}\right)$, Sloan $($ griz), and Wide-field Infrared Survey Explorer $\left(W_{3} W_{4}\right)$ photometric systems. Uncertainties in the photometric magnitudes are not used in the analysis, and reference consistency for each bandpass was maintained where ever possible (see Boyajian et al. 2013 for references and details). Unlike the analysis in Boyajian et al. (2013), we refrain from using Two Micron All Sky Survey $(\mathrm{JHK})$ photometry in our analysis because it is saturated for the majority of these bright stars and thus introduces several percent more uncertainty in the results.

We also adopt the metallicities compiled in Boyajian et al. $(2012 b, 2013)$ in our analysis. It was noted in Boyajian et al. (2012b, 2013) that there is no uniform source of metallicity measurements for the complete sample of interferometrically observed stars in the anthology. This is problematic because although the precision of measuring metallicity within a method is very good ( \pm 0.03 dex; Valenti \& Fischer 2005), the accuracy between references is severely lacking (up to $0.2 \mathrm{dex}$; Torres et al. 2012). Where systematic offsets between different groups using different analysis methods exist, the sensitivity of the data when used for the empirical calibrations is greatly reduced, ultimately compromising the precision of our relations. This absence of a complete and uniform data set prompted Boyajian et al. (2013) to reference the metallicities for the anthology stars from the Anderson \& Francis (2011) catalog, where the values are an average of numerous references available in the literature. This crude approach is currently the best option to unify the data set. However, using the averages makes accurate characterization of the metallicity errors difficult, particularly on an object-to-object basis. For these reasons, we do not include the errors of metallicity in our analysis.

In summary, the sample we use consists of 124 main-sequence stars with measured limb-darkened angular diameters of better than $5 \%$ precision. The sample has spectral types ranging from A to $\mathrm{M}$, with metallicities fairly evenly distributed around solar \pm 0.5 dex. All stars reside in the local neighborhood $(\sim<50 \mathrm{pc})$, thus no correction for reddening was applied to their published photometric magnitudes.

The zero-magnitude angular diameter, $\theta_{m_{\lambda}=0}$ in Equation (1), represents the angular diameter that our star would have if it were at a distance at which its apparent magnitude equals zero (see Section 1); it is thus a wavelength-dependent quantity. It is defined as

$$
\log \theta_{m_{\lambda}=0}=\log \theta_{\mathrm{LD}}+0.2 m_{\lambda},
$$

where $m_{\lambda}$ is the apparent magnitude for a star in filter $\lambda$, and $\theta_{\mathrm{LD}}$ is the angular diameter of the star, corrected for limb-darkening. Full derivations of this equation can be found in di Benedetto (1993), Fouque \& Gieren (1997), Kervella et al. (2004b) and references therein. ${ }^{4}$

Historically, the values for $\theta_{m_{\lambda}=0}$ in different filters were linearly modeled with respect to the broadband color index. These relations, initially developed in Barnes \& Evans (1976) and called the "Barnes-Evans relations,"would, for example, take the form of $a+b X$, where $X$ is a color index $\left(\lambda-\lambda^{\prime}\right)$. More recently, nonlinear functions have preferentially been used to map literature photometry data to predict stellar angular size (e.g., from Bonneau et al. 2006; Kervella \& Fouqué 2008). In order to model the data in this work, we continue the use of a polynomial:

$$
\log \theta_{m_{\lambda}=0}=\sum_{i=0}^{n} a_{i} X^{i}
$$

where $X$ is the observed color index.

\subsection{Application}

\subsubsection{Angular Diameters from Two-band Photometry}

We solve for the results defined by Equation (2) using the nonlinear least-squares fitting function MPFIT . pro (Markwardt 2009) in IDL, weighting the data by the errors in the limbdarkened angular diameters. For every color index we model, we use the resulting $\chi^{2}$ and degrees of freedom to calculate an $F$-value for successive $i$ versus $i+1$ polynomial models in order to determine the statistical significance of the additional parameter. The $F$-test probability is computed using IDL (using MPFTEST.pro, also available in the Markwardt 2009

\footnotetext{
4 Equation (1) is also functionally identical to the definition found in Hindsley \& Bell (1989; Equation (5)), $S_{V}=5 \log \theta+V_{0}$, although this formulation omits the intermediary surface brightness term, $S_{V}$, an issue to be addressed in a forthcoming paper.
} 
suite of IDL routines), where we deem whether the new fit with additional parameters is statistically significant if $<0.05$. Section 2.2.2 introduces metallicity as an additional variable into the equation, and where applicable, those solutions should trump the results derived from Equation (2).

In Table 1, we show the Equation (2) coefficients for all variations of the observable photometric magnitudes and color indices. The total number of stars used in the fit, $N$, deviates from 124 because photometric observations are not available for some stars in some bandpasses. The magnitude range listed in Table 1 applies only to the value of the color index, and these relations should not be extrapolated past these limits. The two last columns of Table 1 give the reduced $\chi^{2}$ and standard deviation of the residuals in percent, calculated by $\operatorname{StdDev}\left[\left(\theta_{m_{\lambda}=0 ; \text { Obs. }}-\theta_{m_{\lambda}=0 ; \text { Calc. }}\right) / \theta_{m_{\lambda}=0 ; \text { Obs. }}\right] \times 100 \%$.

In Figures 2 through 8 , we show the data and the polynomial fits of the solutions with the format of Equation (2), whose coefficients are given in Table 1 . In each plot, the color of the data point reflects the metallicity of the star, as depicted in the legends. The bottom panel in each panel of Figures 2 through 8 shows the fractional residuals of the data to the fit, where the horizontal dotted line marks a zero deviation.

\subsubsection{Metallicity Dependence}

The metal abundance in a star changes the opacity due to line blanketing, and thus changes the total flux emitted at different wavelengths, particularly in the bluest colors. While intermediate-band photometric systems (e.g., Strömgren; Strömgren 1963) have proven to be useful in determining stellar metallicities consistent with spectroscopically derived values, broadband photometric systems are less sensitive to such measurements in comparison (for example, see Árnadóttir et al. 2010 and references therein).

The dependence of surface brightness relations on stellar metallicity has been tested but remains empirically unproven (Kervella \& Fouqué 2008; Kervella et al. 2004b). This is a challenging task with the data at hand because not only are the errors in metallicity large, but there is only a modest range in metallicities within the sample. However, visual inspection of the residuals in the $(B-V)$ model (left hand side of Figure 9) shows a remarkable correlation with metallicity. The top panel of Figure 11 confirms the trend with the residuals with respect to metallicity in the $(B-V)$ model.

As such, we test a model that includes an additional parameter to account for a metallicity dependence in each of the modeled color indices. We choose a multi-variate polynomial function of the form

$$
\log \theta_{m_{\lambda}=0}=\sum_{i=0}^{n} a_{i} X^{i}+b Y,
$$

where stellar color and stellar metallicity $[\mathrm{Fe} / \mathrm{H}]$ are represented by $X$ and $Y$, respectively. We also impose limits restricting the data to exclude $\mathrm{M}$ dwarfs $\left(T_{\text {eff }}<4000 \mathrm{~K}\right)$ because metallicity uncertainties for the latest-type stars are a factor of 10 greater than those for solar-type stars.

To model each color index, we follow the approach described above (Section 2.2.1) by increasing the number of additional parameters in Equation (3) until no improvement is shown in the test statistics. The results for this model are then compared with a model that excludes the last term in Equation (3) to determine the probability of random improvement by including metallicity. Since the magnitude of line blanketing is a known function of stellar temperature (Sandage 1969), we also used this method to test whether adding a cross-term parameter (i.e., $c X Y$ ) to Equation (3) would change the results of the analysis and found that it did not.

We detect a statistically significant metallicity dependence in the $(B-V)$ and the $(g-r)$ color indices, which is no surprise since these colors are the shortest filter wavelengths included in this analysis, and thus the most influenced by changes in metallicity. We find that the $(B-V)$ surface brightness relation has an extremely significant solution ( $p$-value of $\sim 10^{-18}$ ) when including metallicity as an additional parameter. This solution is explicitly expressed in Equation (4). It is restricted to be valid for stars with $-0.02<(B-V)<0.95$, has a reduced $\chi^{2}=23.5$ (total of $N=100$ stars), and has a standard deviation of the residuals of $4.5 \%$ :

$$
\begin{aligned}
\log \theta_{m_{V}=0}= & (0.52005 \pm 0.00121) \\
& +(0.90209 \pm 0.01348)(B-V) \\
& +(-0.67448 \pm 0.03676)(B-V)^{2} \\
& +(0.39767 \pm 0.02611)(B-V)^{3} \\
& +(-0.08476 \pm 0.00161)[\mathrm{Fe} / \mathrm{H}]
\end{aligned}
$$

The analysis of the Sloan $(g-r)$ colors leads to a calculated $p$-value of $\sim 10^{-5}$, a detection much less outstanding than the $(B-V)$ color, but still a quite significant improvement. The metallicity-dependent form of the relation is expressed by Equation (5). The fit uses $N=66$ stars, has a reduced $\chi^{2}=25.5$, and the standard deviation of the residuals is $5.8 \%$ :

$$
\begin{aligned}
\log \theta_{m_{V}=0}= & (0.66645 \pm 0.00138) \\
& +(0.74459 \pm 0.00315)(g-r) \\
& +(-0.08276 \pm 0.00346)[\mathrm{Fe} / \mathrm{H}] .
\end{aligned}
$$

In Figures 9 and 10, we show side by side the solutions with and without metallicity for the $(B-V)$ and $(g-r)$ colors. Figure 11 shows the trends detected in the surface brightness relation residuals with respect to the star's metallicity. This improvement when including metallicity in the model argues that the inclusion of metallicity cannot be ignored when using the $(B-V)$ or $(g-r)$ color indices. This is the first time such a dependence on metallicity has been found from empirical data on surface brightness relations (see the discussion in Section 3).

\section{DISCUSSION}

We compare our results to the works from van Belle (1999), Bonneau et al. (2006), and Kervella \& Fouqué (2008), all of which use predictions based on empirical values to determine stellar angular sizes.

The van Belle (1999) calibration for main-sequence stars uses a limited data set based on Hanbury Brown et al. (1974a) — which was all that was available at the time — to derive their calibrations. This data set consisted of 11 main-sequence B- and A-type stars $(-0.5 \lesssim(V-K) \lesssim+0.5)$, and is extrapolated to the Sun's position at $(V-K) \simeq 1.5$. We plot their $(V-K)$ relation (green triple dot-dashed line) in Figure 6. This shows only a slight systematic offset of the van Belle (1999) relation to smaller diameters; however, considering the small sample size used in the calibration, this offset is statistically insignificant with the quoted $1 \sigma$ errors of the relation in van Belle (1999).

The Bonneau et al. (2006) work, also referred to as The JMMC Stellar Diameters Catalog or JSDC, ${ }^{5}$ is the backbone of The

\footnotetext{
5 http://www.jmmc.fr/catalogue_jsdc.htm
} 
Table 1

Solutions to Angular Diameter Relations

\begin{tabular}{|c|c|c|c|c|c|c|c|c|c|c|}
\hline$\lambda$ & $\begin{array}{l}\text { Color } \\
\text { Index }\end{array}$ & $\begin{array}{l}\text { No. of } \\
\text { Points }\end{array}$ & $\begin{array}{l}\text { Range } \\
(\mathrm{mag})\end{array}$ & $a_{0} \pm \sigma$ & $a_{1} \pm \sigma$ & $a_{2} \pm \sigma$ & $a_{3} \pm \sigma$ & $a_{4} \pm \sigma$ & $\begin{array}{c}\text { Reduced } \\
\chi^{2}\end{array}$ & $\begin{array}{c}\sigma \\
(\%) \\
\end{array}$ \\
\hline$V$ & $(B-V)^{\dagger}$ & 124 & {$[-0.02-1.73]$} & $0.49612 \pm 0.00111$ & $1.11136 \pm 0.00939$ & $-1.18694 \pm 0.02541$ & $0.91974 \pm 0.02412$ & $-0.19526 \pm 0.00738$ & 73.8 & 7.8 \\
\hline$V$ & $\left(V-R_{J}\right)$ & 81 & [0.00-1.69] & $0.49743 \pm 0.00100$ & $0.82790 \pm 0.00304$ & $-0.04227 \pm 0.00196$ & & $\ldots$ & 60.2 & 7.0 \\
\hline$V$ & $\left(V-I_{J}\right)$ & 80 & {$[-0.03-2.69]$} & $0.51435 \pm 0.00101$ & $0.45435 \pm 0.00349$ & $0.04152 \pm 0.00390$ & $-0.02124 \pm 0.00108$ & $\cdots$ & 45.4 & 5.9 \\
\hline$V$ & $\left(V-R_{C}\right)$ & 34 & {$[-0.01-1.24]$} & $0.50524 \pm 0.00109$ & $1.31557 \pm 0.00788$ & $-0.49134 \pm 0.01850$ & $0.26584 \pm 0.01131$ & $\ldots$ & 28.1 & 4.9 \\
\hline$V$ & $\left(V-I_{C}\right)$ & 34 & {$[-0.02-2.77]$} & $0.50659 \pm 0.00103$ & $0.56448 \pm 0.00793$ & $0.17460 \pm 0.01647$ & $-0.16268 \pm 0.01002$ & $0.03292 \pm 0.00184$ & 25.7 & 5.1 \\
\hline$V$ & $\left(V-R_{K}\right)$ & 64 & {$[-0.21-1.32]$} & $0.70071 \pm 0.00056$ & $0.93899 \pm 0.00252$ & $-0.09351 \pm 0.00229$ & $\cdots$ & $\cdots$ & 170.0 & 7.7 \\
\hline$V$ & $\left(V-I_{K}\right)$ & 64 & {$[-0.33-2.42]$} & $0.67424 \pm 0.00060$ & $0.58321 \pm 0.00150$ & $-0.05227 \pm 0.00073$ & $\ldots$ & $\cdots$ & 67.5 & 5.3 \\
\hline$V$ & $(V-J)$ & 95 & {$[-0.12-4.24]$} & $0.52464 \pm 0.00086$ & $0.38167 \pm 0.00108$ & $-0.01431 \pm 0.00026$ & $\ldots$ & $\cdots$ & 41.3 & 5.1 \\
\hline$V$ & $(V-H)$ & 86 & {$[-0.13-4.77]$} & $0.53019 \pm 0.00059$ & $0.27917 \pm 0.00030$ & $\ldots$ & $\ldots$ & $\ldots$ & 45.4 & 5.3 \\
\hline$V$ & $(V-K)$ & 97 & {$[-0.15-5.04]$} & $0.53246 \pm 0.00057$ & $0.26382 \pm 0.00028$ & $\ldots$ & $\cdots$ & $\ldots$ & 34.2 & 4.6 \\
\hline$V$ & $(V-W 3)$ & 44 & {$[0.76-5.50]$} & $0.57935 \pm 0.00188$ & $0.23879 \pm 0.00054$ & $\ldots$ & $\ldots$ & $\ldots$ & 8.6 & 5.1 \\
\hline$V$ & $(V-W 4)$ & 111 & {$[0.03-5.62]$} & $0.52073 \pm 0.00214$ & $0.28979 \pm 0.00283$ & $-0.01641 \pm 0.00112$ & $0.00144 \pm 0.00013$ & $\cdots$ & 20.9 & 7.2 \\
\hline$g$ & $(g-r)$ & 79 & {$[-0.23-1.40]$} & $0.66728 \pm 0.00203$ & $0.58135 \pm 0.01180$ & $0.88293 \pm 0.03470$ & $-1.41005 \pm 0.04331$ & $0.67248 \pm 0.01736$ & 155.3 & 9.7 \\
\hline$g$ & $(g-i)$ & 79 & {$[-0.43-2.78]$} & $0.69174 \pm 0.00125$ & $0.54346 \pm 0.00266$ & $-0.02149 \pm 0.00097$ & $\ldots$ & $\ldots$ & 111.1 & 9.2 \\
\hline$g$ & $(g-z)$ & 79 & {$[-0.58-3.44]$} & $0.72292 \pm 0.00108$ & $0.46563 \pm 0.00203$ & $-0.02499 \pm 0.00061$ & $\ldots$ & $\cdots$ & 123.1 & 9.5 \\
\hline$g$ & $(g-J)$ & 60 & {$[-0.02-5.06]$} & $0.52662 \pm 0.00226$ & $0.34439 \pm 0.00216$ & $-0.00920 \pm 0.00039$ & $\ldots$ & $\ldots$ & 19.4 & 4.9 \\
\hline$g$ & $(g-H)$ & 53 & [0.75-5.59] & $0.46684 \pm 0.00571$ & $0.36437 \pm 0.00667$ & $-0.04206 \pm 0.00229$ & $0.00493 \pm 0.00024$ & $\ldots$ & 23.1 & 4.7 \\
\hline$g$ & $(g-K)$ & 60 & {$[-0.01-5.86]$} & $0.51356 \pm 0.00443$ & $0.29555 \pm 0.00506$ & $-0.02164 \pm 0.00171$ & $0.00272 \pm 0.00018$ & $\ldots$ & 20.7 & 4.2 \\
\hline$R_{J}$ & $\left(R_{J}-J\right)$ & 74 & {$[-0.12-1.86]$} & $0.54161 \pm 0.00081$ & $0.44407 \pm 0.00370$ & $0.11255 \pm 0.00668$ & $-0.06697 \pm 0.00278$ & $\ldots$ & 50.9 & 5.6 \\
\hline$R_{J}$ & $\left(R_{J}-H\right)$ & 66 & {$[-0.13-2.80]$} & $0.53572 \pm 0.00066$ & $0.31753 \pm 0.00058$ & $\ldots$ & $\ldots$ & $\cdots$ & 53.2 & 5.5 \\
\hline$R_{J}$ & $\left(R_{J}-K\right)$ & 75 & {$[-0.15-3.06]$} & $0.53954 \pm 0.00063$ & $0.29108 \pm 0.00052$ & $\ldots$ & $\ldots$ & $\cdots$ & 37.2 & 4.6 \\
\hline$R_{J}$ & $\left(R_{J}-W 4\right)$ & 74 & {$[0.03-3.56]$} & $0.53243 \pm 0.00161$ & $0.30816 \pm 0.00236$ & $-0.01557 \pm 0.00067$ & $\cdots$ & $\cdots$ & 23.0 & 5.9 \\
\hline$R_{C}$ & $\left(R_{C}-J\right)$ & 27 & {$[-0.11-3.00]$} & $0.53356 \pm 0.00093$ & $0.44321 \pm 0.00178$ & $-0.02541 \pm 0.00063$ & $\ldots$ & $\cdots$ & 49.0 & 4.7 \\
\hline$R_{C}$ & $\left(R_{C}-H\right)$ & 26 & {$[-0.12-3.53]$} & $0.53563 \pm 0.00075$ & $0.30231 \pm 0.00048$ & $\ldots$ & $\ldots$ & $\cdots$ & 62.3 & 5.6 \\
\hline$R_{C}$ & $\left(R_{C}-K\right)$ & 27 & {$[-0.14-3.80]$} & $0.53282 \pm 0.00075$ & $0.28271 \pm 0.00045$ & $\ldots$ & $\cdots$ & $\cdots$ & 45.7 & 4.7 \\
\hline$R_{C}$ & $\left(R_{C}-W 4\right)$ & 30 & {$[0.20-4.38]$} & $0.56261 \pm 0.00110$ & $0.24575 \pm 0.00050$ & $\cdots$ & $\cdots$ & $\cdots$ & 32.2 & 6.3 \\
\hline$R_{K}$ & $\left(R_{K}-J\right)$ & 59 & {$[0.09-2.58]$} & $0.47966 \pm 0.00129$ & $0.49606 \pm 0.00227$ & $-0.03434 \pm 0.00087$ & $\ldots$ & $\cdots$ & 105.6 & 5.8 \\
\hline$R_{K}$ & $\left(R_{K}-H\right)$ & 57 & {$[0.08-3.17]$} & $0.51110 \pm 0.00077$ & $0.31285 \pm 0.00050$ & $\cdots$ & $\cdots$ & $\cdots$ & 78.8 & 5.3 \\
\hline$R_{K}$ & $\left(R_{K}-K\right)$ & 61 & {$[0.06-3.43]$} & $0.51325 \pm 0.00076$ & $0.29002 \pm 0.00046$ & $\ldots$ & $\ldots$ & $\ldots$ & 53.7 & 4.5 \\
\hline$R_{K}$ & $\left(R_{K}-W 4\right)$ & 52 & {$[0.17-3.93]$} & $0.46844 \pm 0.00520$ & $0.38664 \pm 0.00902$ & $-0.05846 \pm 0.00479$ & $0.00748 \pm 0.00077$ & $\cdots$ & 19.8 & 5.4 \\
\hline$I_{J}$ & $\left(I_{J}-J\right)$ & 75 & {$[-0.09-0.78]$} & $0.56179 \pm 0.00061$ & $0.80862 \pm 0.00204$ & $\ldots$ & $\cdots$ & $\ldots$ & 99.1 & 9.1 \\
\hline$I_{J}$ & $\left(I_{J}-H\right)$ & 66 & {$[-0.10-1.37]$} & $0.53904 \pm 0.00086$ & $0.36206 \pm 0.00270$ & $0.03569 \pm 0.00201$ & $\cdots$ & $\cdots$ & 69.9 & 6.6 \\
\hline$I_{J}$ & $\left(I_{J}-K\right)$ & 75 & {$[-0.12-1.63]$} & $0.53510 \pm 0.00066$ & $0.35175 \pm 0.00088$ & $\ldots$ & $\ldots$ & $\ldots$ & 42.9 & 5.2 \\
\hline$I_{J}$ & $\left(I_{J}-W 4\right)$ & 74 & [0.04-2.13] & $0.53766 \pm 0.00171$ & $0.34918 \pm 0.00392$ & $-0.02832 \pm 0.00180$ & $\ldots$ & $\ldots$ & 27.5 & 6.6 \\
\hline$I_{C}$ & $\left(I_{C}-J\right)$ & 27 & {$[-0.10-1.47]$} & $0.55013 \pm 0.00074$ & $0.54738 \pm 0.00117$ & $\ldots$ & $\cdots$ & $\ldots$ & 58.1 & 5.6 \\
\hline$I_{C}$ & $\left(I_{C}-H\right)$ & 26 & {$[-0.11-2.00]$} & $0.53026 \pm 0.00077$ & $0.36595 \pm 0.00079$ & $\cdots$ & $\cdots$ & $\cdots$ & 97.6 & 7.4 \\
\hline$I_{C}$ & $\left(I_{C}-K\right)$ & 27 & {$[-0.13-2.27]$} & $0.52800 \pm 0.00077$ & $0.32919 \pm 0.00071$ & $\cdots$ & $\cdots$ & $\cdots$ & 64.9 & 5.4 \\
\hline$I_{C}$ & $\left(I_{C}-W 4\right)$ & 30 & {$[0.14-2.85]$} & $0.55786 \pm 0.00114$ & $0.27009 \pm 0.00076$ & $\cdots$ & $\cdots$ & $\cdots$ & 30.5 & 6.4 \\
\hline$I_{K}$ & $\left(I_{K}-J\right)$ & 59 & [0.21-1.48] & $0.44692 \pm 0.00097$ & $0.59745 \pm 0.00125$ & $\cdots$ & $\cdots$ & $\cdots$ & 146.4 & 7.8 \\
\hline$I_{K}$ & $\left(I_{K}-H\right)$ & 57 & [0.20-2.07] & $0.48163 \pm 0.00090$ & $0.37123 \pm 0.00078$ & $\cdots$ & $\cdots$ & $\cdots$ & 113.7 & 7.1 \\
\hline$I_{K}$ & $\left(I_{K}-K\right)$ & 61 & [0.18-2.33] & $0.49053 \pm 0.00087$ & $0.33156 \pm 0.00069$ & $\cdots$ & $\cdots$ & $\cdots$ & 73.8 & 5.5 \\
\hline$I_{K}$ & $\left(I_{K}-W 4\right)$ & 52 & {$[0.23-2.83]$} & $0.50028 \pm 0.00345$ & $0.33757 \pm 0.00484$ & $-0.01992 \pm 0.00145$ & $\ldots$ & $\ldots$ & 23.6 & 5.9 \\
\hline
\end{tabular}

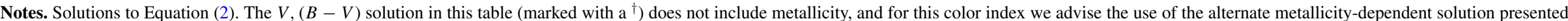
in the text (Equation (4)); refer to Section 2 for details. 

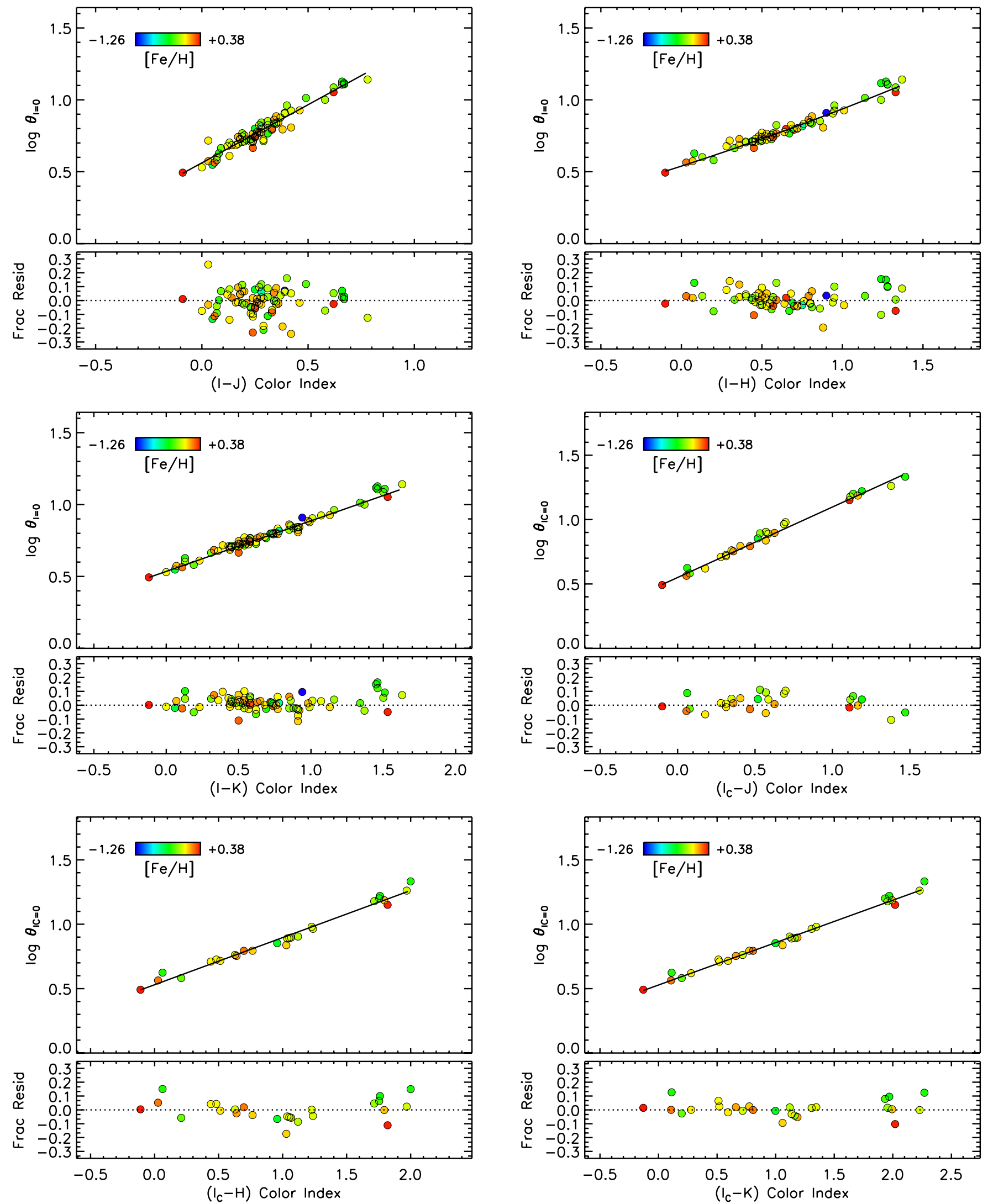

Figure 2. Top panel shows the zero-magnitude limb-darkened angular diameter plotted against the color index. The solid black line plots the polynomial relation for the region in which the relation holds true (Table 1). The color of the data point reflects the metallicity of the star as depicted in the legend. The bottom panel shows the fractional residuals to our fit $\left(\theta_{\text {Obs. }}-\theta_{\text {Fit }}\right) / \theta_{\text {Obs. }}$, where the dotted line indicates zero deviation, see Section 2 for details.

(A color version of this figure is available in the online journal.) 

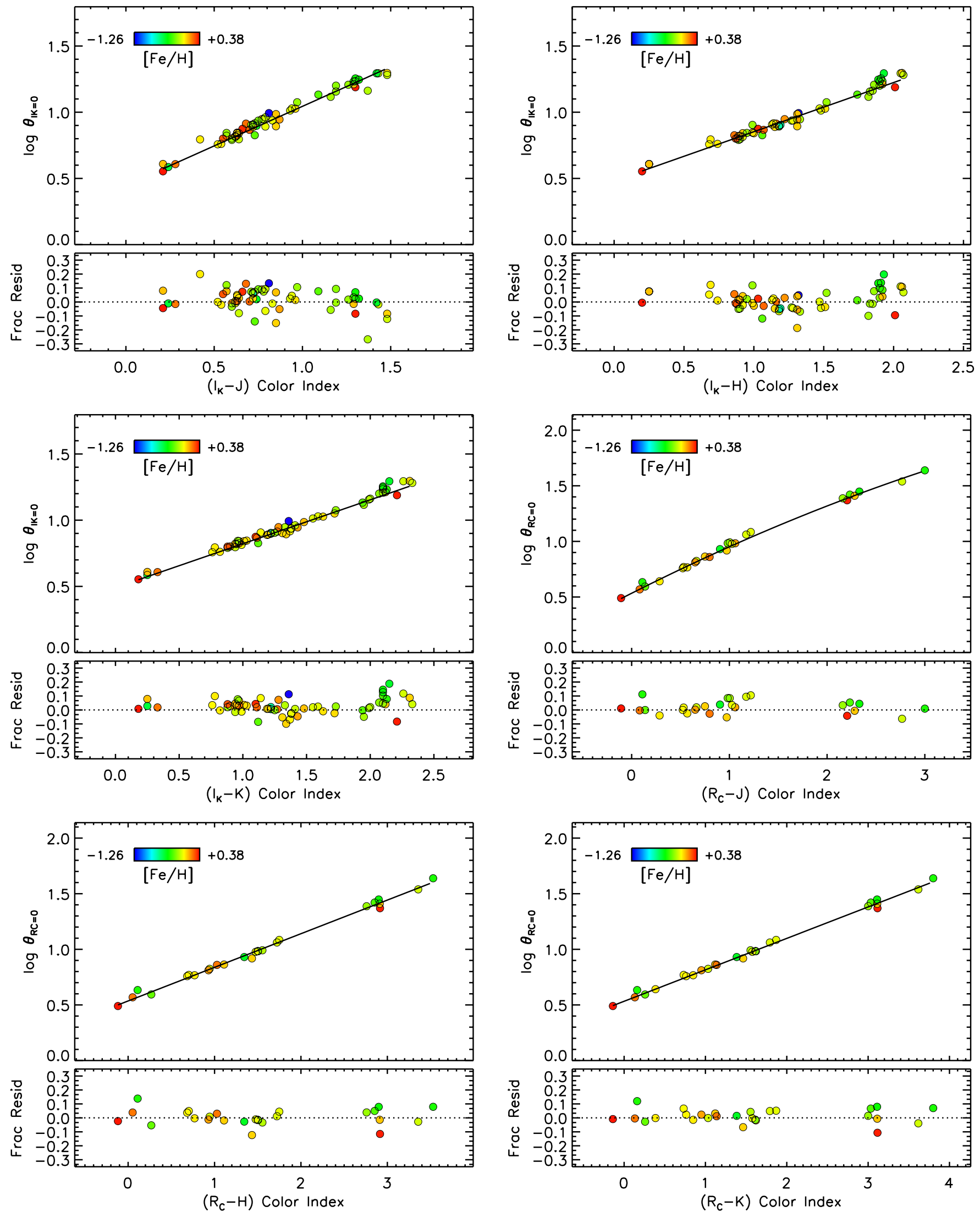

Figure 3. Top panel shows the zero-magnitude limb-darkened angular diameter plotted against the color index. The solid black line plots the polynomial relation for the region in which the relation holds true (Table 1). The color of the data point reflects the metallicity of the star as depicted in the legend. The bottom panel shows the fractional residuals to our fit $\left(\theta_{\text {Obs. }}-\theta_{\text {Fit }}\right) / \theta_{\text {Obs. }}$, where the dotted line indicates zero deviation; see Section 2 for details.

(A color version of this figure is available in the online journal.) 

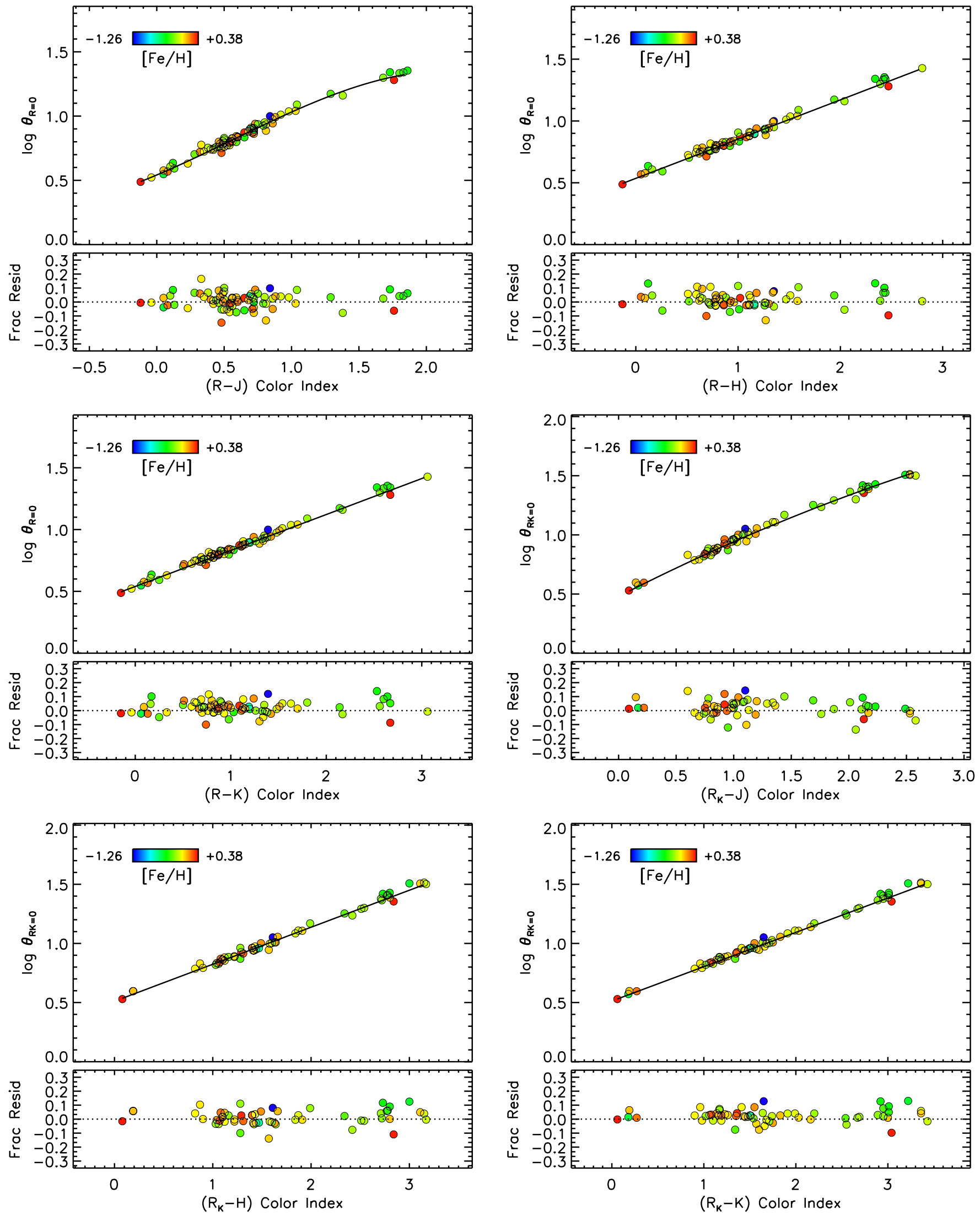

Figure 4. Top panel shows the zero-magnitude limb-darkened angular diameter plotted against the color index. The solid black line plots the polynomial relation for the region in which the relation holds true (Table 1). The color of the data point reflects the metallicity of the star as depicted in the legend. The bottom panel shows the fractional residuals to our fit $\left(\theta_{\text {Obs. }}-\theta_{\text {Fit }}\right) / \theta_{\text {Obs. }}$, where the dotted line indicates zero deviation; see Section 2 for details.

(A color version of this figure is available in the online journal.) 

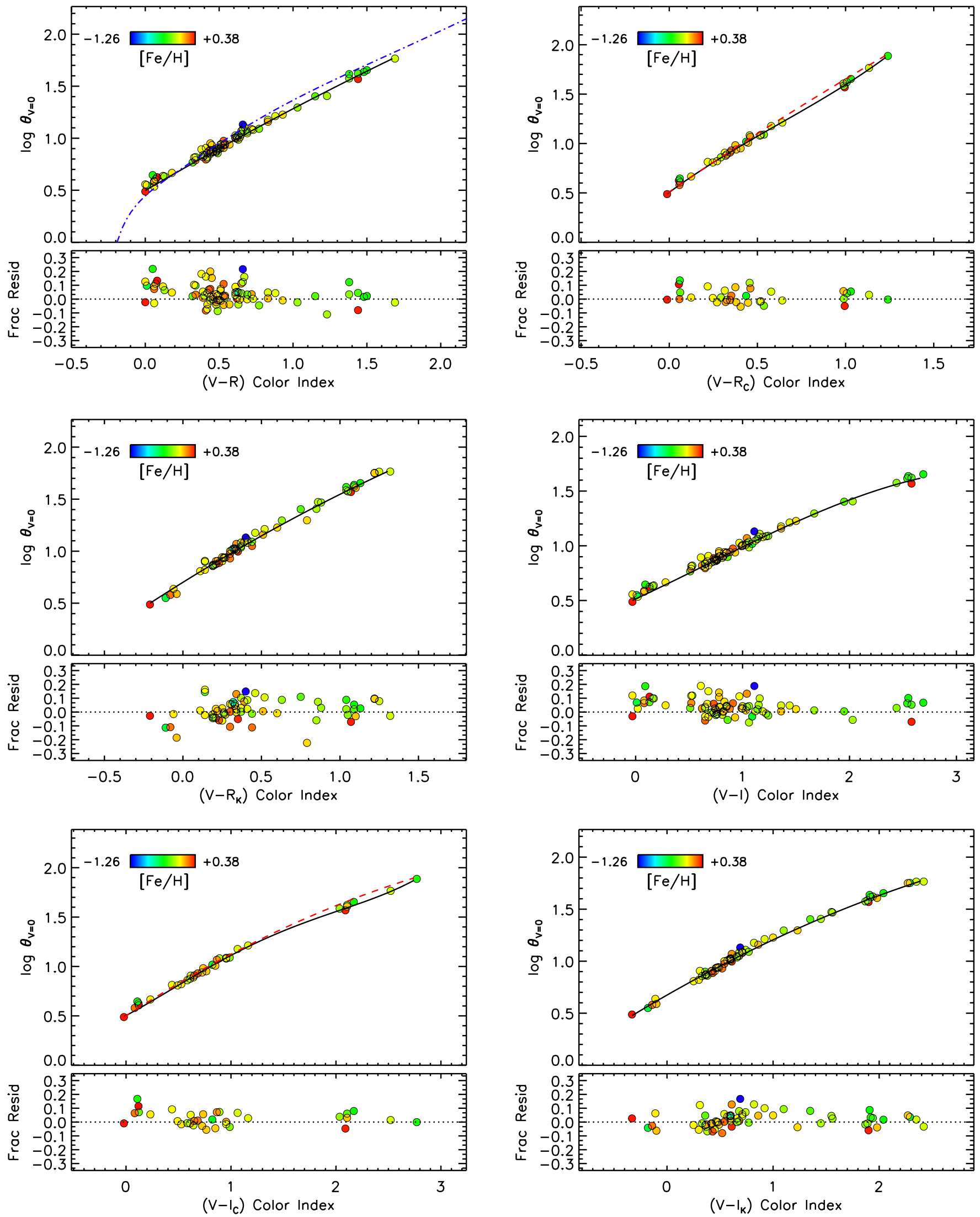

Figure 5. Top panel shows the zero-magnitude limb-darkened angular diameter plotted against the color index. The solid black line plots the polynomial relation for the region in which the relation holds true (Table 1). The color of the data point reflects the metallicity of the star as depicted in the legend. A solution from Kervella \& Fouqué (2008) is plotted as a dashed red line, and a solution from Bonneau et al. (2006) is plotted as a blue dash-dotted line. The bottom panel shows the fractional residuals to our fit $\left(\theta_{\text {Obs. }}-\theta_{\text {Fit }}\right) / \theta_{\text {Obs. }}$, where the dotted line indicates zero deviation; see Section 2 for details.

(A color version of this figure is available in the online journal.) 

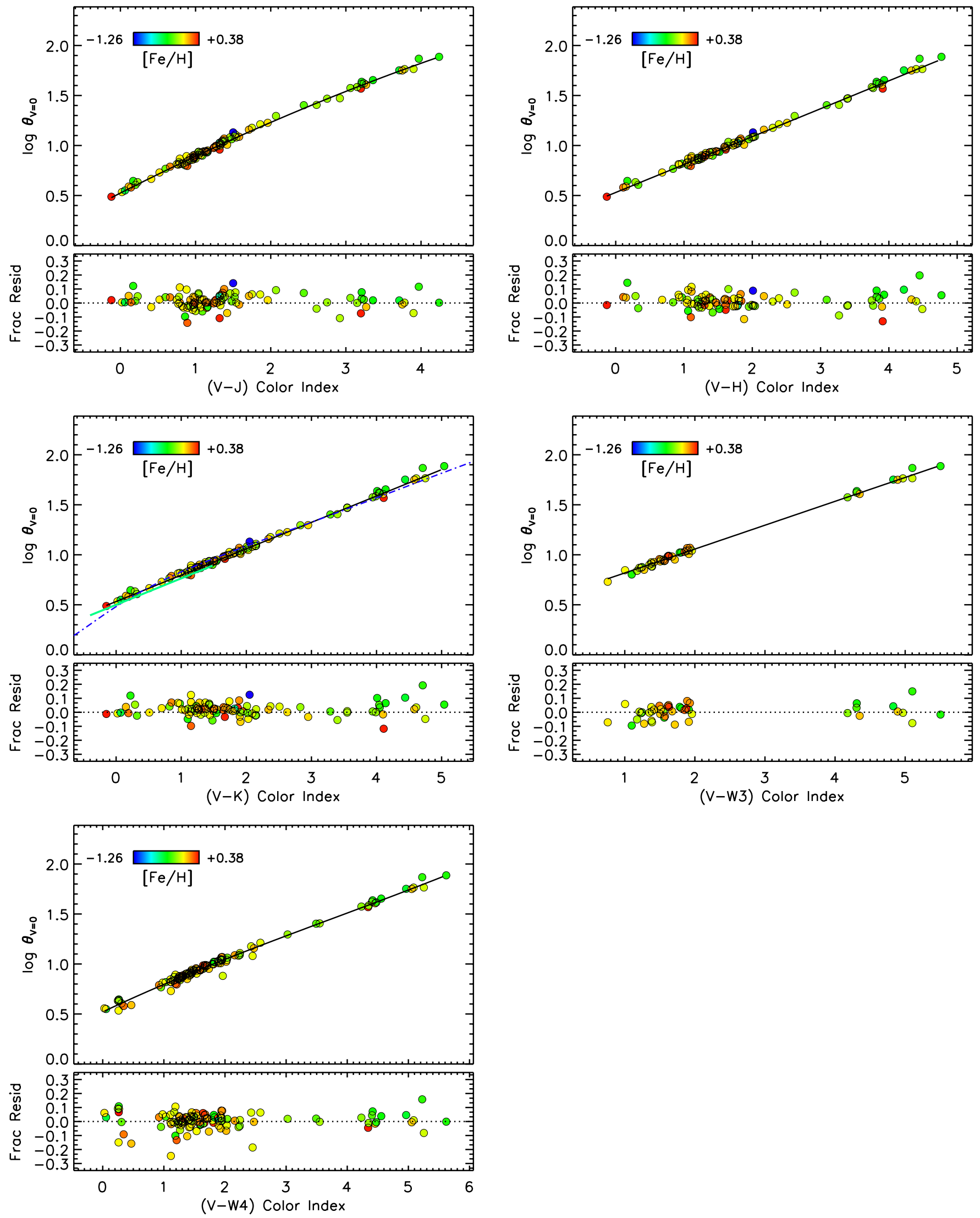

Figure 6. Top panel shows the zero-magnitude limb-darkened angular diameter plotted against the color index. The solid black line plots the polynomial relation for the region in which the relation holds true (Table 1). The color of the data point reflects the metallicity of the star as depicted in the legend. A solution from Bonneau et al. (2006) is plotted as a blue dash-dotted line. A solution from van Belle (1999) is plotted as a green triple dot-dashed line. The bottom panel shows the fractional residuals to our fit $\left(\theta_{\text {Obs. }}-\theta_{\text {Fit }}\right) / \theta_{\text {Obs. }}$, where the dotted line indicates zero deviation; see Section 2 for details.

(A color version of this figure is available in the online journal.) 

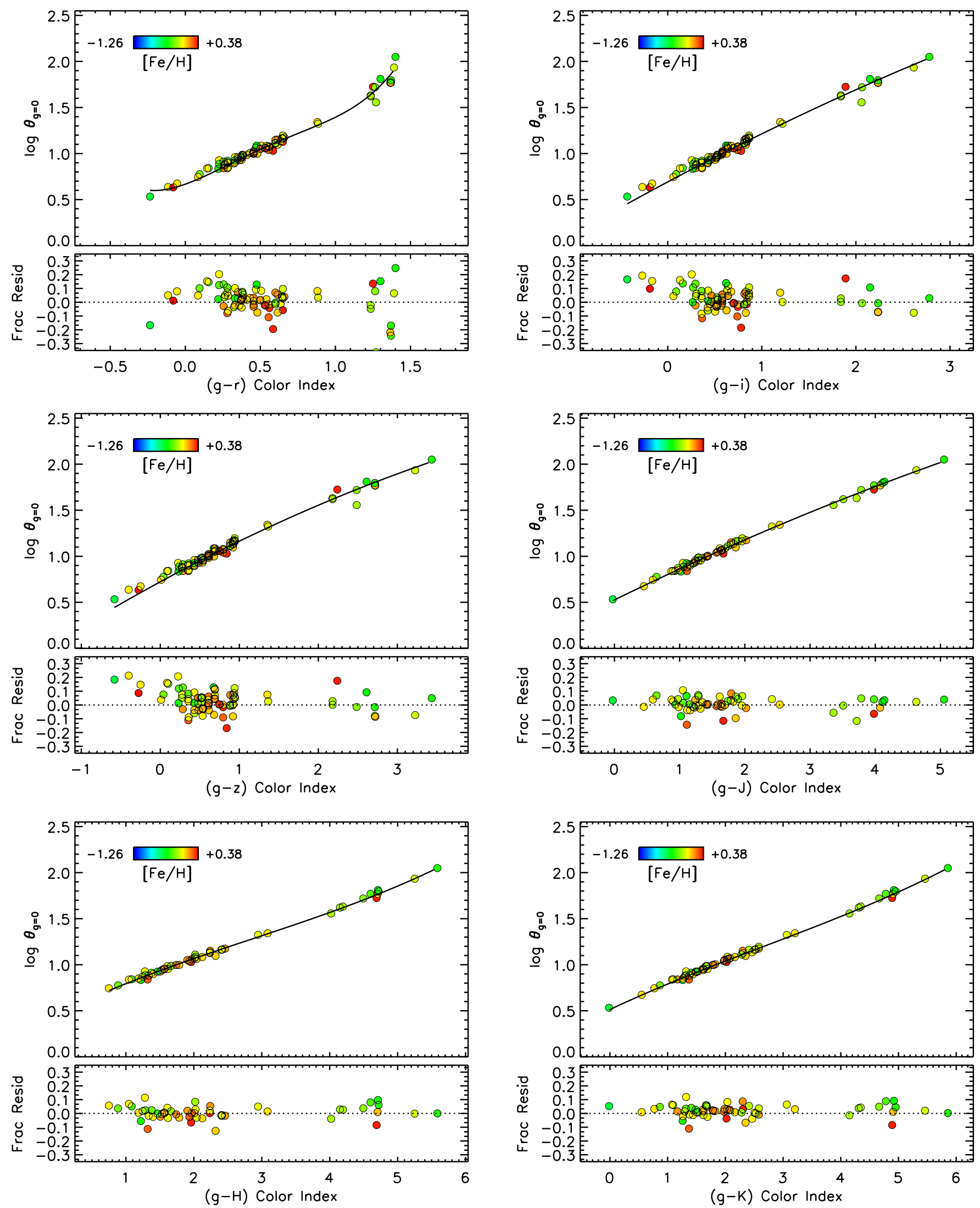

Figure 7. Top panel shows the zero-magnitude limb-darkened angular diameter plotted against the color index. The solid black line plots the polynomial relation for the region in which the relation holds true (Table 1). The color of the data point reflects the metallicity of the star as depicted in the legend. The bottom panel shows the fractional residuals to our fit $\left(\theta_{\text {Obs. }}-\theta_{\text {Fit }}\right) / \theta_{\text {Obs. }}$, where the dotted line indicates zero deviation; see Section 2 for details.

(A color version of this figure is available in the online journal.) 

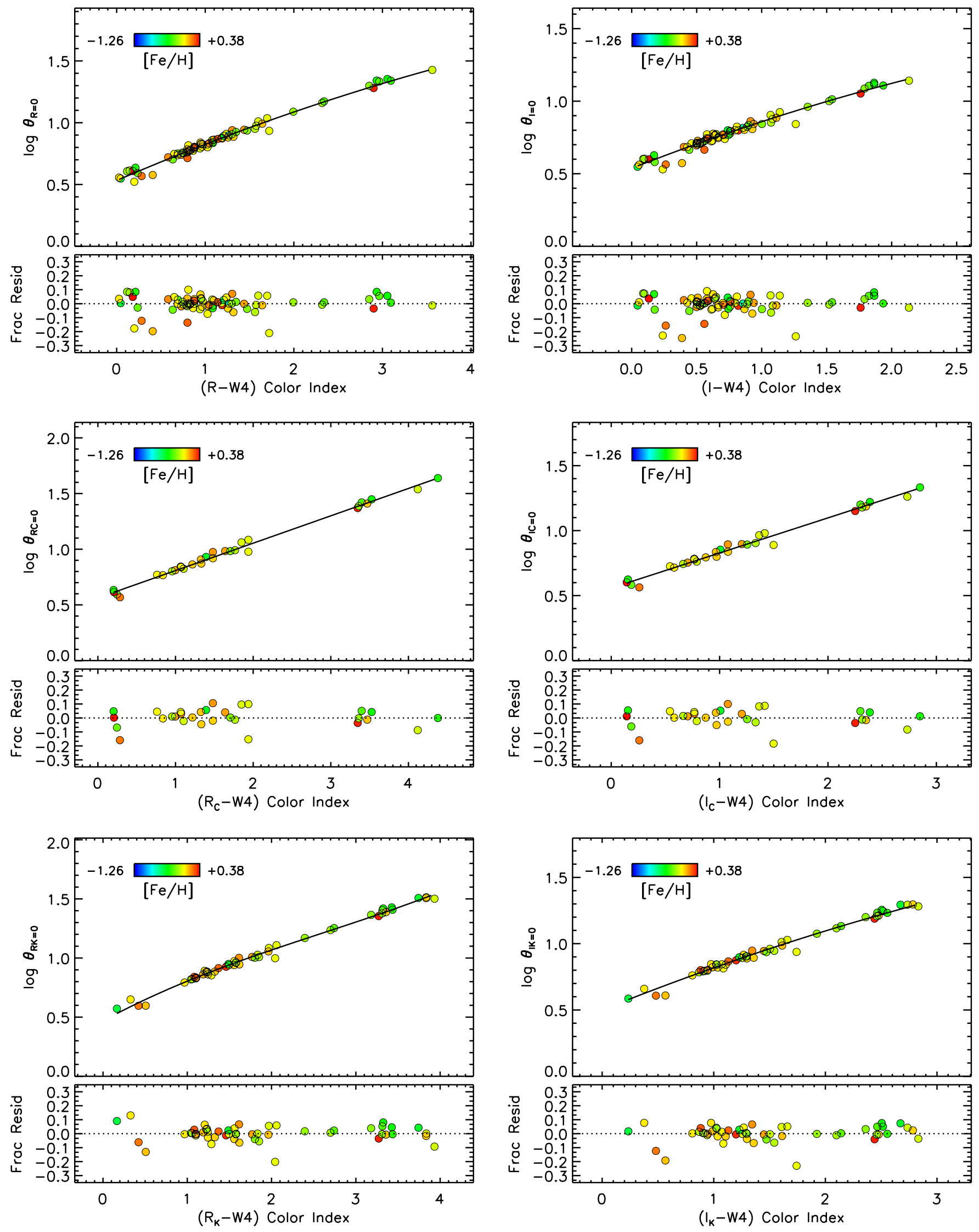

Figure 8. Top panel shows the zero-magnitude limb-darkened angular diameter plotted against the color index. The solid black line plots the polynomial relation for the region in which the relation holds true (Table 1). The color of the data point reflects the metallicity of the star as depicted in the legend. A solution from Kervella $\&$ Fouqué (2008) is plotted as a dashed red line. The bottom panel shows the fractional residuals to our fit $\left(\theta_{\text {Obs. }}-\theta_{\text {Fit }}\right) / \theta_{\text {Obs. }}$, where the dotted line indicates zero deviation; see Section 2 for details.

(A color version of this figure is available in the online journal.) 

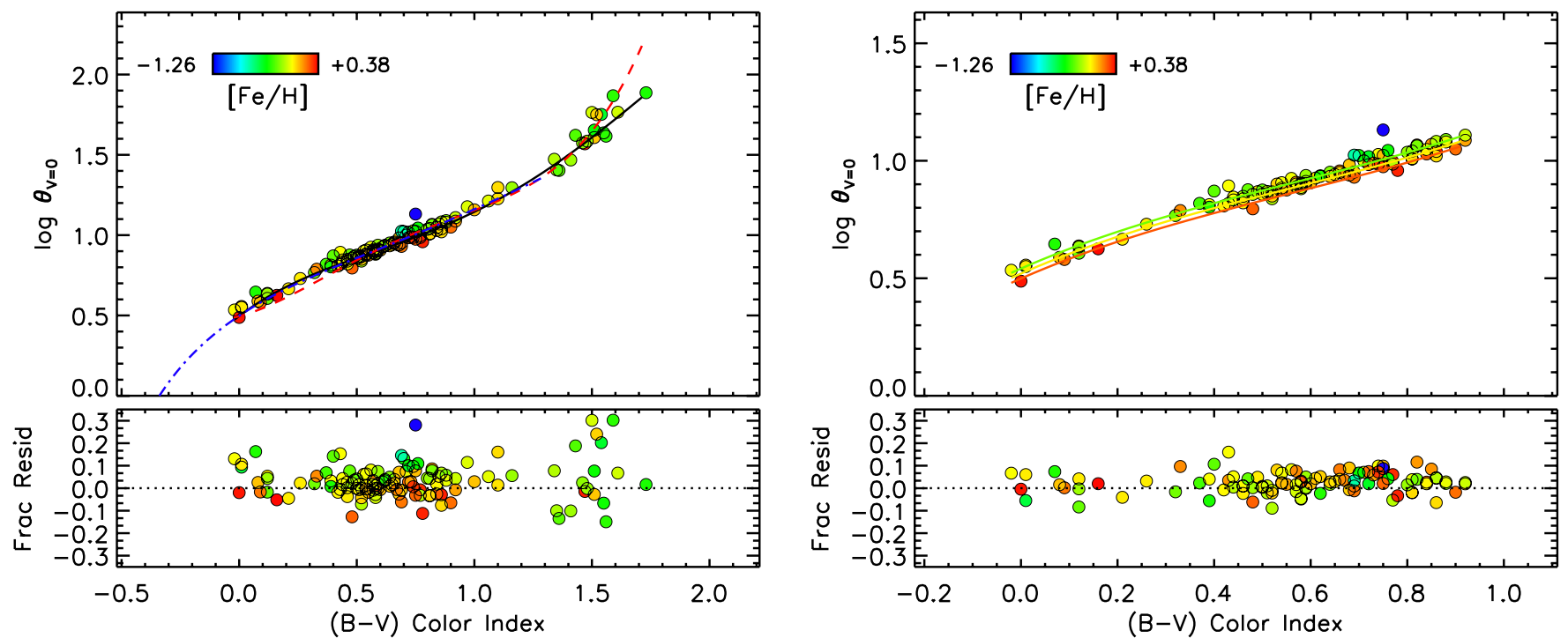

Figure 9. Top panels show the zero-magnitude limb-darkened angular diameter plotted against the $(B-V)$ color index. The plot on the left is the metallicity-independent solution where the solid black line plots the polynomial relation for the region in which the relation holds true (Equation (2), Table 1). A solution from Kervella \& Fouque (2008) is plotted as a dashed red line, and the solution from Bonneau et al. (2006) is plotted as a blue dash-dotted line. The plot on the right shows the solution for the metallicity-dependent solution (Equation (4)), where lines of constant metallicity are shown for $[\mathrm{Fe} / \mathrm{H}]=+0.25,0.0$, and -0.25 dex (red, yellow, and green, respectively). In all plots, the color of the data point reflects the metallicity of the star as depicted in the legend. The bottom panel shows the fractional residuals to our fits $\left(\theta_{\text {Obs. }}-\theta_{\text {Fit }}\right) / \theta_{\text {Obs. }}$, where the dotted line indicates zero deviation; see Figure 11 for a close-up view on the comparison of the residuals. See Sections 2 and 3 for details and a discussion.

(A color version of this figure is available in the online journal.)
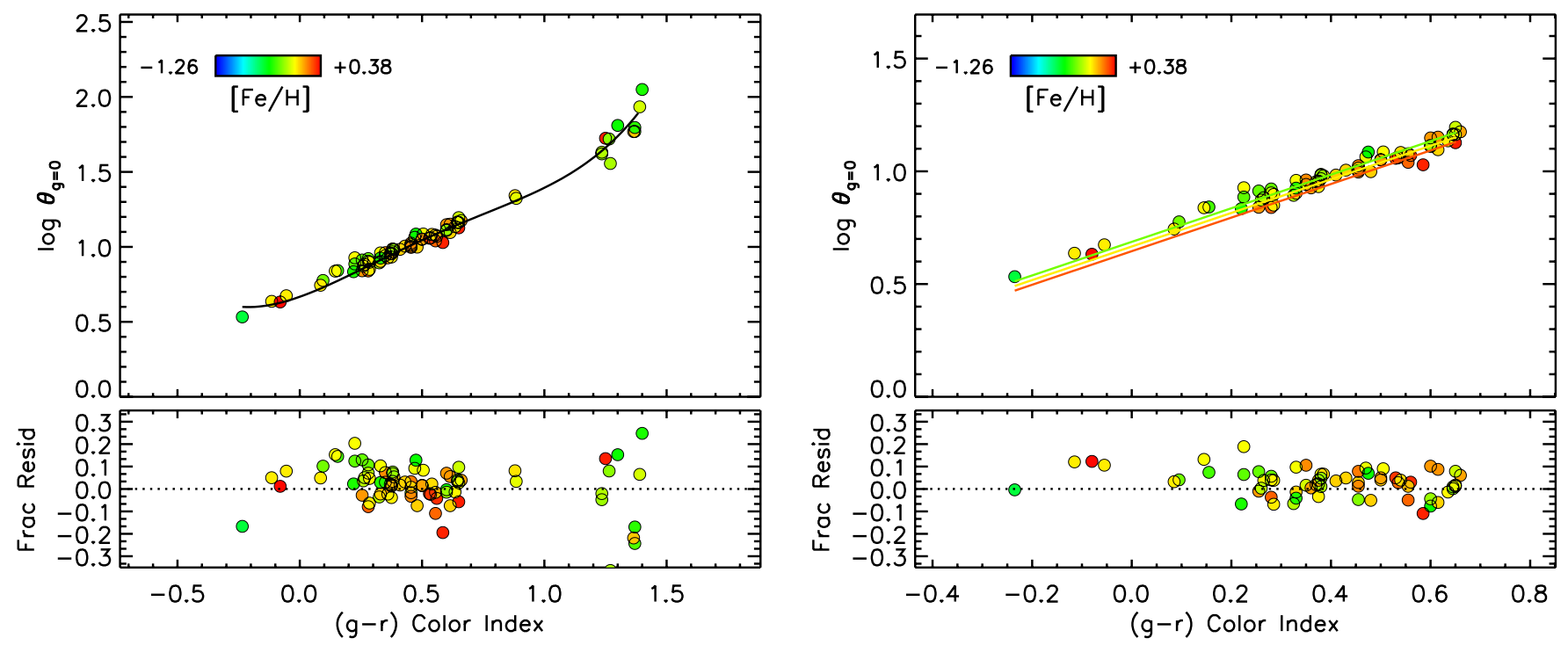

Figure 10. Top panels show the zero-magnitude limb-darkened angular diameter plotted against the $(g-r)$ color index. The plot on the left is the metallicity-independent solution where the solid black line plots the polynomial relation for the region in which the relation holds true (Equation (2), Table 1). The plot on the right shows the solution for the metallicity-dependent solution (Equation (5)), where lines of constant metallicity are shown for $[\mathrm{Fe} / \mathrm{H}]=+0.25,0.0$, and -0.25 dex (red, yellow, and green, respectively). In all plots, the color of the data point reflects the metallicity of the star as depicted in the legend. The bottom panel shows the fractional residuals to our fits $\left(\theta_{\text {Obs. }}-\theta_{\text {Fit }}\right) / \theta_{\text {Obs. }}$, where the dotted line indicates zero deviation; see Figure 11 for a close-up view on the comparison of the residuals with respect to metallicity. See Sections 2 Section 3 for details and a discussion.

(A color version of this figure is available in the online journal.)

SearchCal catalog, managed by the JMMC working group. ${ }^{6}$ It is a tool to allow an observer to search for suitable interferometric calibrators in an interface tailored to their individual needs. The application allows for the observable photometric colors and estimated angular sizes to be used in conjunction with observatory and instrument configurations in order to derive instrumental visibility estimates for each source. The method used to derive the predicted angular diameters is outlined and

\footnotetext{
6 http://www.jmmc.fr/searchcal_page.htm
}

defined in Bonneau et al. (2006). To calibrate the Bonneau et al. (2006) relations, a broad source of data available from eclipsing binaries, interferometric measurements, and lunar occultations are used (Barnes et al. 1978; Andersen 1991; Ségransan et al. 2003; Mozurkewich et al. 2003). The Bonneau et al. (2006) calibration data cover the full extent of luminosity classes ( $\mathrm{V}$ to I) and spectral types (O to $\mathrm{M})$. They use fourth-order polynomials to model the data for the $(B-V)$ and $(V-K)$ relations, and a fifth-order polynomial to model the data for $(V-R)$, where the solutions to their fits yield uncertainties 

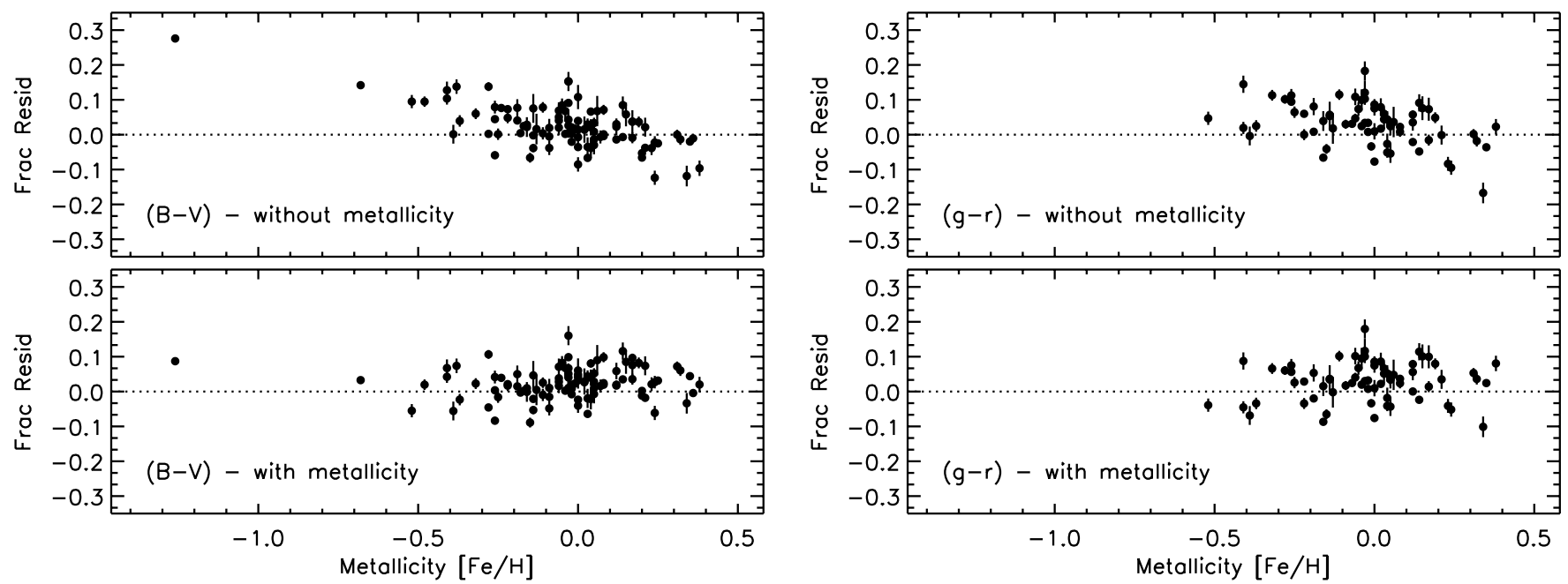

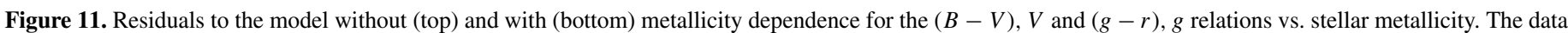
and fit for each solution are shown in Figures 9 and 10; see Sections 2.2.1, 2.2.2, and 3 for details.

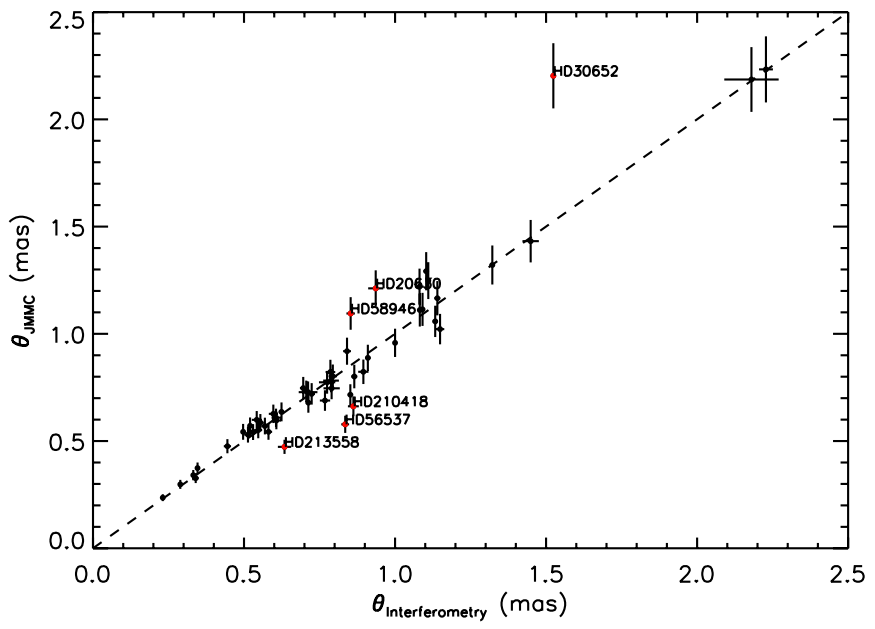

Figure 12. Interferometric angular diameters compared with those in the JMMC Catalog (Bonneau et al. 2006), showing agreement of $\theta_{\text {Interferometry }} / \theta_{\text {JMMC }}=$ $0.999 \pm 0.095$. The dashed line shows a 1:1 agreement. We show stars with differences greater than $3 \sigma$ in red and label their Henry Draper (HD) numbers in the plot window; see Section 3 for details.

(A color version of this figure is available in the online journal.)

of $8 \%, 10 \%$, and $7 \%$ for $(B-V),(V-R)$, and $(V-K)$, respectively.

We show the $(B-V),(V-R)$, and $(V-K)$ model fits from Bonneau et al. (2006) along with our data in Figures 9, 5, and 6 , respectively (blue dash-dotted line). The $(B-V)$ and $(V-K)$ data and models show that the Bonneau et al. (2006) solution is indistinguishable from the one presented here. Major differences in our model and the Bonneau et al. (2006) model are only relevant within the $(V-R)$ relations. We suspect that this is possibly due to the Ducati (2002) $R$-band photometric systems used in Bonneau et al. (2006) not being identical to the Johnson $R$-band used here. In Figure 12, we show a direct comparison of the interferometrically measured angular diameters to the final angular diameters in the JMMC Catalog. We find that $\theta_{\text {Interferometry }} / \theta_{\text {JMMC }}=0.999 \pm 0.095$, undoubtedly excellent agreement.

The Kervella \& Fouqué (2008) relations are calibrated using interferometrically measured stellar angular diameters. Similar to our sample selection, they use only stars with diameters measured with better than $5 \%$ precision, which reside on or near the main sequence. At the time Kervella \& Fouqué (2008) was published, the sample size available for calibrating their relations consisted of 34 stars. Since then, major advances in the field of interferometry have made it possible for several interferometric diameter surveys of nearby stars to contribute to the field (e.g., van Belle \& von Braun 2009; Boyajian et al. 2012a, 2013; Huber et al. 2012). Aside from the advantage of statistical improvement by virtue of possessing a larger sample size, we are afforded the luxury of being able to treat the data for our analysis in a variety of ways without sacrificing significance. For instance, Kervella \& Fouqué (2008) consider repeated angular diameter measurements of the same star as unique sources. In order to avoid any bias in our results, we are able to use the weighted averages presented in the Boyajian et al. (2012b, 2013) compilations, if multiple measurements exist. ${ }^{7}$

We show the Kervella \& Fouqué $(2008)(B-V),\left(V-R_{C}\right)$, and $\left(V-I_{C}\right)$ relations in Figures 9 and 5 (red dashed line). The Kervella \& Fouqué (2008) $\left(V-R_{C}\right)$ and $\left(V-I_{C}\right)$ relations are consistent with our own for stars earlier than $\sim \mathrm{K} 0$ $\left(\left(V-R_{C}\right) \lesssim 0.7\right.$, and $\left.\left(V-I_{C}\right) \lesssim 1.2\right)$, but overestimate diameters by $\sim 10 \%$ for the latest-type stars, a difference of $\sim 2 \sigma$. The Kervella \& Fouqué (2008) $(B-V)$ relation compared to the one presented here, as well as the one in Bonneau et al. (2006), differs for stars earlier than $\sim \mathrm{F} 3$ (or $(B-V) \lesssim 0.35$ ), by $\sim 15 \%$, also $\sim 2 \sigma$. Stars later than $\sim \mathrm{M} 0$ (or $(B-V) \gtrsim 1.5$ ) also show disagreement between our solution and Kervella \& Fouqué (2008); however, these differences can be attributed to the sparse sampling of data used in Kervella \& Fouqué (2008) with respect to the color index, especially on the endpoints of the relation, as well as the increased amount of scatter in the full data set used in both calibrations.

Nonetheless, compared to the Kervella \& Fouqué (2008) treatment, our solutions do not improve the precision of the (metallicity-independent) surface brightness relations. Specifically, the scatter in both $(B-V)$ relations yields just below $8 \%$, although the sample size is different: $n=124$ and $n=42$ for this work and Kervella \& Fouqué (2008), respectively. The $\left(V-R_{\mathrm{C}}\right)$ and $\left(V-I_{\mathrm{C}}\right)$ relations show

\footnotetext{
7 If we considered every radius measurement an independent quantity, a sample size of $n=137$ stars would be available for analysis. The averaging of repeated sources brings this sample size down to $n=124$ stars.
} 
contrasting improvement with the new data added to the sample: $\sigma\left(V-R_{\mathrm{C}}\right)=4.9 \%, 4.5 \%$ and $\sigma\left(V-I_{\mathrm{C}}\right)=5.1 \%, 5.6 \%$ for this work and Kervella \& Fouqué (2008), respectively. However, we note that the inclusion of metallicity in our $(B-V)$ model knocks the scatter down to $4.5 \%$.

We detect a significant dependence of the stellar metallicity on the $(B-V)$ and $(g-r)$ surface brightness relations. But why was this not detected before now? The linear surface brightness relations in Kervella et al. (2004b) showed no correlation with metallicity on the visible to infrared calibrations $((B-L)$; see their Figure 5). Our detection is a likely consequence of several factors.

1. Metallicities of the 29 stars used in the Kervella et al. (2004b) calibrations come from nine different references; concerns with systematics between data sets are relevant at this level of inconsistency.

2. Over the past decade, M-dwarf metallicities have been refined using more sophisticated techniques and calibrations. In fact, all metallicities for the M dwarfs cited in Kervella et al. (2004b) have been refined, exceeding differences greater than 1.0 dex. The $\mathrm{M}$ dwarfs comprise $25 \%$ of the 29 stars in the Kervella et al. (2004b) sample and they use these $\mathrm{M}$ dwarfs to draw their conclusions on metallicity. Our decision to reject $\mathrm{M}$ dwarfs decreases our sample size by two dozen (also 25\%); however, we have a healthy sized sample remaining $(N=100)$ to draw statistically significant results.

3. The visual-to-infrared $(B-L)$ color index is not sensitive to metallicity.

4. The sample size has increased by a factor of four since the Kervella et al. (2004b) calibrations, allowing for statistically robust analysis.

The more recent, nonlinear calibrations using visible photometry presented in Kervella \& Fouqué (2008) did not explore the possible impact of metallicity on the relations, but it was suggested to the reader that it was expected to be small compared to the intrinsic dispersion of the relations. Our solutions for $(B-V)$ and $(g-r)$ show that this is not the case, and even though the typical metallicity of our sample is close to solar with a dispersion of \pm 0.5 dex, including metallicity in these solutions decreases the scatter of the fits to a level similar to those that do not show an $[\mathrm{Fe} / \mathrm{H}]$ dependence (Table 1). We are undoubtedly helped by the larger sample size, as well as a more consistent method of treating the data available for this work for such a clear trend to become apparent in these two color indices.

It is still the case that we are not able to obtain much better than $5 \%$ precision in the surface brightness relations, even with the inclusion of metallicity as an additional parameter. We attempted two further sieves of the data where we rejected all stars with angular diameter errors $>2 \%$ and all with errors $>1 \%$, leaving a total of $N=86$ and $N=40$ calibration stars, respectively. Unfortunately, in no case does this improve the $\chi^{2}$ nearly enough to be statistically significant below a $p$-value of $\sim 0.99$ when compared to the full data set. This is expected, however, since the fits we present are weighted to the errors in angular diameter. At this point, it is unclear whether the large reduced $\chi^{2}$ 's of the fits are astrophysical in nature, or purely measurement error in observed metallicities and/or photometric magnitudes. The importance of a complete and uniform database of these calibration star stellar properties cannot be understated.

\section{SUMMARY AND CONCLUSION}

In this paper, we take advantage of a large data set of directly measured, interferometric stellar radii to calibrate surface brightness relations that are able to predict angular sizes of stars through photometry in two bands. We provide the polynomial coefficients for each model in Table 1 for a large number of commonly used broadband colors. All solutions and data are shown in Figures 2 through 8. Compared to previously published work on this topic, we find generally good agreement in the calculated relations with some exceptions, as we discuss in Section 3 . We show that the $(B-V)$ and $(g-r)$ colors show a clear improvement when including metallicity as an extra term (Figures 9 and 10; Equations (4) and (2)). This is the first time surface brightness relations have shown a dependence on stellar metallicity from empirical data (see the discussion in Section 3).

The well-understood procedure to obtain multi-band photometry on stars that are much too faint and small for their radii to be measured interferometrically enables a relatively straightforward and empirical prediction of their angular diameters. The formulations presented here expand the parameter space of the color-radius relations in Boyajian et al. (2012b) beyond lowmass $\mathrm{K}$ and $\mathrm{M}$ dwarfs because they are insensitive to stellar evolution. Thus, with the knowledge of a star's trigonometric parallax values, this work provides access to physical radii for the full span of A-M type stars.

T.S.B. acknowledges support provided through NASA grant ADAP12-0172. G.v.B. is supported in part through NASA grant NNX13AF01G, and NSF grant AST-1212203. The authors would like to thank Nicolas Nardetto, Jean-Baptiste Le Bouquin, and Pierre Kervella for useful discussions on the topic. This research has made use of the Jean-Marie Mariotti Center SearchCal service ${ }^{8}$ co-developed by FIZEAU and LAOG/ IPAG, and of the CDS Astronomical Databases SIMBAD and VIZIER. ${ }^{9}$ This research has made use of the SIMBAD literature database, operated at CDS, Strasbourg, France, and of NASA's Astrophysics Data System. This research has made use of the VizieR catalog access tool, CDS, Strasbourg, France. This publication makes use of data products from the Widefield Infrared Survey Explorer, which is a joint project of the University of California, Los Angeles, and the Jet Propulsion Laboratory/California Institute of Technology, funded by the National Aeronautics and Space Administration.

\section{REFERENCES}

Anderson, E., \& Francis, C. 2011, yCat, 5137, 0

Andersen, J. 1991, A\&ARv, 3, 91

Árnadóttir, A. S., Feltzing, S., \& Lundström, I. 2010, A\&A, 521, A40

Assef, R. J., Gaudi, B. S., \& Stanek, K. Z. 2009, ApJ, 701, 1616

Baines, E. K., McAlister, H. A., ten Brummelaar, T. A., et al. 2008, ApJ, 680, 728

Baines, E. K., White, R. J., Huber, D., et al. 2012, ApJ, 761, 57

Barnes, T. G., \& Evans, D. S. 1976, MNRAS, 174, 489

Barnes, T. G., Evans, D. S., \& Moffett, T. J. 1978, MNRAS, 183, 285

Bazot, M., Ireland, M. J., Huber, D., et al. 2011, A\&A, 526, L4

Bigot, L., Kervella, P., Thévenin, F., \& Ségransan, D. 2006, A\&A, 446, 635

Bigot, L., Mourard, D., Berio, P., et al. 2011, A\&A, 534, L3

Bonneau, D., Clausse, J.-M., Delfosse, X., et al. 2006, A\&A, 456, 789

Boyajian, T. S., McAlister, H. A., Baines, E. K., et al. 2008, ApJ, 683, 424

Boyajian, T. S., McAlister, H. A., Cantrell, J. R., et al. 2009, ApJ, 691, 1243

Boyajian, T. S., McAlister, H. A., van Belle, G., et al. 2012a, ApJ, 746, 101

Boyajian, T. S., von Braun, K., van Belle, G., et al. 2012b, ApJ, 757, 112

\footnotetext{
8 Available at http://www.jmmc.fr/searchcal.

9 Available at http://cdsweb.u-strasbg.fr/.
} 
Boyajian, T. S., von Braun, K., van Belle, G., et al. 2013, ApJ, 771, 40

Calchi Novati, S., Dall'Ora, M., Gould, A., et al. 2010, ApJ, 717, 987

Chiavassa, A., Bigot, L., Kervella, P., et al. 2012, A\&A, 540, A5

Claret, A. 2000, A\&A, 363, 1081

Crepp, J. R., Johnson, J. A., Fischer, D. A., et al. 2012, ApJ, 751, 97

Davis, J., Ireland, M. J., North, J. R., et al. 2011, PASA, 28, 58

di Benedetto, G. P. 1993, A\&A, 270, 315

di Benedetto, G. P. 1995, ApJ, 452, 195

di Benedetto, G. P. 1998, A\&A, 339, 858

Di Benedetto, G. P. 2005, MNRAS, 357, 174

Di Folco, E., Thévenin, F., Kervella, P., et al. 2004, A\&A, 426, 601

Ducati, J. R. 2002, yCat, 2237, 0

Fouque, P., \& Gieren, W. P. 1997, A\&A, 320, 799

Groenewegen, M. A. T. 2007, A\&A, 474, 975

Hanbury Brown, R., Davis, J., \& Allen, L. R. 1974a, MNRAS, 167, 121

Hanbury Brown, R. H., Davis, J., Lake, R. J. W., \& Thompson, R. J. 1974b, MNRAS, 167, 475

Hindsley, R. B., \& Bell, R. A. 1989, ApJ, 341, 1004

Huber, D., Ireland, M. J., Bedding, T. R., et al. 2012, ApJ, 760, 32

Kervella, P., Bersier, D., Mourard, D., et al. 2004a, A\&A, 428, 587

Kervella, P., \& Fouqué, P. 2008, A\&A, 491, 855

Kervella, P., Thévenin, F., Di Folco, E., \& Ségransan, D. 2004b, A\&A, 426, 297

Kervella, P., Thévenin, F., Morel, P., Bordé, P., \& Di Folco, E. 2003a, A\&A, 408, 681

Kervella, P., Thévenin, F., Morel, P., et al. 2004c, A\&A, 413, 251

Kervella, P., Thévenin, F., Ségransan, D., et al. 2003b, A\&A, 404, 1087
Lacy, C. H. 1977, ApJ, 213, 458

Ligi, R., Mourard, D., Lagrange, A. M., et al. 2012, A\&A, 545, A5

Markwardt, C. B. 2009, in ASP Conf. Ser. 411, Astronomical Data Analysis Software and Systems XVIII, ed. D. A. Bohlender, D. Durand, \& P. Dowler (San Francisco: CA: ASP), 251

Mozurkewich, D., Armstrong, J. T., Hindsley, R. B., et al. 2003, AJ, 126,2502

Nordgren, T. E., Germain, M. E., Benson, J. A., et al. 1999, AJ, 118, 3032

Nordgren, T. E., Sudol, J. J., \& Mozurkewich, D. 2001, AJ, 122, 2707

Sandage, A. 1969, ApJ, 158, 1115

Ségransan, D., Kervella, P., Forveille, T., \& Queloz, D. 2003, A\&A, 397, L5

Southworth, J., Maxted, P. F. L., \& Smalley, B. 2005, A\&A, 429, 645

Storm, J., Gieren, W., Fouqué, P., et al. 2011a, A\&A, 534, A94

Storm, J., Gieren, W., Fouqué, P., et al. 2011b, A\&A, 534, A95

Strömgren, B. 1963, QJRAS, 4, 8

Thévenin, F., Kervella, P., Pichon, B., et al. 2005, A\&A, 436, 253

Torres, G., Andersen, J., \& Giménez, A. 2010, A\&ARv, 18, 67

Torres, G., Fischer, D. A., Sozzetti, A., et al. 2012, ApJ, 757, 161

Valenti, J. A., \& Fischer, D. A. 2005, ApJS, 159, 141

van Belle, G. T. 1999, PASP, 111, 1515

van Belle, G. T., Lane, B. F., Thompson, R. R., et al. 1999, AJ, 117, 521

van Belle, G. T., \& von Braun, K. 2009, ApJ, 694, 1085

von Braun, K., Boyajian, T. S., Kane, S. R., et al. 2011, ApJL, 729, L26

von Braun, K., Boyajian, T. S., Kane, S. R., et al. 2012, ApJ, 753, 171

Wang, J.-H., Protopapas, P., Chen, W.-P., et al. 2010, AJ, 139, 2003

Wesselink, A. J. 1969, MNRAS, 144, 297 